\title{
A CRISPR-engineered swine model of COL2A1 deficiency recapitulates altered early skeletal developmental defects in humans
}

\author{
Boyan Zhang ${ }^{1}$, Chenyu Wang ${ }^{2}$, Yue Zhang², Yuan Jiang ${ }^{3}$, Yanguo Qin ${ }^{1}$, Daxin Pang ${ }^{4}$ \\ Guizhen Zhang ${ }^{1}$, He Liu ${ }^{1}$, Zicong Xie ${ }^{3}$, Hongming Yuan ${ }^{4}$, Hongsheng Ouyang ${ }^{4}$, Jincheng \\ Wang ${ }^{1}$, and Xiaochun Tang ${ }^{4}$ \\ ${ }^{1}$ Jilin University Second Hospital \\ ${ }^{2}$ Jilin University First Hospital \\ ${ }^{3}$ College of Animal Sciences, Jilin University \\ ${ }^{4}$ Jilin University
}

May 5, 2020

\begin{abstract}
Loss-of-function mutations in the COL2A1 gene were recently described as a cause of type II collagenopathy, a major subgroup of genetic skeletal diseases. However, the pathogenic mechanisms associated with COL2A1 mutations remain unclear, and there are few large-mammal models of these diseases. In this study, we established a swine model carrying COL2A1 mutations using CRISPR/Cas9 and somatic cell nuclear transfer technologies. Animals mutant for COL2A1 exhibited severe skeletal dysplasia characterized by shortened long bones, abnormal vertebrae, depressed nasal bridge, and cleft palate. Importantly, COL2A1 mutant piglets suffered tracheal collapse, which was almost certainly the cause of their death shortly after birth. In conclusion, we have demonstrated for the first time that overt and striking skeletal dysplasia occurring in human patients can be recapitulated in large transgenic mammals. This model underscores the importance of employing large animals as models to investigate the pathogenesis and potential therapeutics of skeletal diseases.
\end{abstract}

\section{Keywords}

COL2A1 mutation, CRISPR/Cas9, swine model, genetic skeletal diseases, cartilage development, tracheal collapse

\section{Introduction}

Genetic skeletal disorders (GSDs) arise through mutations that disrupt normal skeletal development and homeostasis(C. Chen et al., 2016). Although individually rare, GSDs are of important clinical relevance as a group owing to their high overall frequency(Kornak \& Mundlos, 2003). The rapid development of genetic diagnostic technology over the past few decades has led to the identification of 436 GSDs, which have been classified into 42 groups(Mortier et al., 2019). The type II collagen group, one of the major GSD groups identified, consists of 17 clinical disorders that are associated with mutations in the COL2A1 gene, and comprise a spectrum of autosomal-dominant disorders characterized by skeletal dysplasia(Deng, Huang, \& Yuan, 2016; Kannu, Bateman, \& Savarirayan, 2012). Type II collagen is the most abundant protein in cartilage, and, together with additional tissue-specific collagens and proteoglycans, provides structural integrity to this tissue(Myllyharju \& Kivirikko, 2004). Type II collagen, a fibrillar collagen, is a molecule composed of homotrimers of collagen alpha 1 type II $(\alpha 1[\mathrm{II}])_{3}$ chains containing characteristic repeating Gly-X-Y sequences that fold into a triple-helical structure. Although most COL2A1 mutations are found in the triple-helical region, mutations have also been identified in the $\mathrm{N}$ - and C-propeptide domains situated at the amino- and carboxyl-terminal ends of the triple-helical region(Barat-Houari et al., 2016). 
Genetic disorders resulting from COL2A1 mutations, known as type II collagenopathies, include a spectrum of highly variable chondrodysplasias that are characterized according to severity. These include mild types like avascular necrosis of the femoral head (ANFH) and Stickler syndrome type I (STL 1); severe types like spondyloepiphyseal dysplasia congenita (SEDC) and Kniest dysplasia; and lethal types like achondrogenesis type II (ACG 2) and platyspondylic skeletal dysplasia, Torrance type (PLSD-T)(Deng et al., 2016). Although 663 independent probands previously reported with 460 different COL2A1 variants have been identified that may be associated with different phenotypes(Zhang et al., 2020), the causes of skeletal deformities and the underlying pathomechanisms remain unclear. To address this, several genetically modified mouse models have been established and characterized. For example, pronounced short-limbed dwarfism was developed in a mouse model with deletion of Col2a1 exon 7, as well as in transgenic mice expressing the Col2a1p.G574S mutation(Maddox, Garofalo, Smith, Keene, \& Horton, 1997; Metsaranta et al., 1992). Mild phenotypes, such as the ocular features found in STL 1, have been observed in Col2a1 ${ }^{+/-}$mice(Kaarniranta et al., 2006). Moreover, a mouse model of SEDC displaying respiratory failure, short limb bones, and a short vertebral column has also been established(Barbieri et al., 2003; Donahue et al., 2003; Macdonald et al., 2013). The frequently lethal phenotypes associated with ACG 2 and PLSD-T were also seen in mouse models with p.R789C and p.D1469A Col2a1 mutations, respectively(Furuichi et al., 2011; Gaiser et al., 2002). Although mouse models carrying Col2a1 mutations show delayed skeletal development and abnormal cartilage composition and structure, they lack the overt and striking skeletal dysplasia observed in larger mammals owing to the limited size of their bones and joints.

Pigs have been widely employed in biological studies owing to their genetic, anatomical, and physiological similarities with humans(Prather, Lorson, Ross, Whyte, \& Walters, 2013). Many genetically engineered pig models have been developed for biomedical research into the underlying mechanisms of human diseases(Holm, Alstrup, \& Luo, 2016; Yan et al., 2018). However, relatively few studies investigating GSDs have employed large mammals, including the pig. The articular structure of the major joints of pigs is more like that of humans when compared with those of smaller model organisms. Furthermore, the tools currently available for genetic manipulation, in combination with somatic cell nuclear transfer (SCNT) technology, allow for the modification of endogenous pig genes and the development of a variety of pig models of human diseases(Han et al., 2017; Yang et al., 2017; Zhou et al., 2015).

In this study, we used CRISPR/Cas 9 to modify the pig COL2A1 gene and establish a swine model of a lethal type II collagenopathy that resembles PLSD-T. The generation of a swine model carrying COL2A1 mutations, which displayed typical skeletal deformities and abnormal cartilage development, demonstrates that large mammals can recapitulate overt and striking skeletal dysplasia caused by genetic variants. Importantly, investigation of the trachea of COL2A1 mutant piglets provided convincing evidence that tracheal collapse was the most likely cause of the death of the mutant piglets shortly after birth. Our findings support that genetically modified swine models are valuable tools for studying the pathogenesis of GSDs and the associated mechanisms.

\section{Materials and Methods}

Ethical statement.

All animal studies were approved by the Animal Welfare and Research Ethics Committee of Jilin University, and all procedures were conducted strictly in accordance with the Guide for the Care and Use of Laboratory Animals. All experimental pigs were obtained from the Huichang Animal Husbandry Science and Technology Co., Ltd. All animal operations were performed under anesthesia, and every effort was made to minimize animal suffering.

sgRNA design and CRISPR/Cas9 vector construction.

The targeted sgRNAs in Figure 1C were designed according to a previously described method (http://crispr.mit.edu/)(Cong et al., 2013). The sequences of the two sgRNA are provided in Figure 1C. The pX330U6-Chimeric_BB-CBh-hSpCas9 plasmid was purchased from Addgene (\#42230). Two complementary sgRNA oligonucleotides with appropriate adaptors were synthesized and then annealed in standard 
Taq buffer (NEB, Beijing, China). The resultant double-stranded DNA was ligated to the BbsI sites of the vector backbone to form the targeting plasmid.

Isolation of porcine fetal fibroblasts and cell culture.

Porcine fetal fibroblasts were derived from 32-day-old Bama miniature pig fetuses. Fetuses were disaggregated in culture medium containing $200 \mathrm{U} / \mathrm{mL}$ collagenase IV (type IV, $260 \mathrm{U} / \mathrm{mg}$; Gibco, Grand Island, NY, USA), $18 \%$ fetal bovine serum (Gibco), $0.0125 \mathrm{mg} / \mathrm{mL}$ DNase I (2,000 U/mg; Sigma, St. Louis, MO, USA), and $1 \%$ penicillin/streptomycin (Gibco) for 5-6 h. Isolated PFFs were resuspended and cultured in 10-cm Petri dishes until subconfluence, and then cultured in Dulbecco's modified Eagle's medium (Gibco) supplemented with $12 \%$ fetal bovine serum. First-generation cells were frozen in fetal bovine serum containing $10 \%$ dimethyl sulfoxide.

Porcine fetal fibroblast transfection and selection.

Approximately $3 \times 10^{6} \mathrm{PFFs}$ were electroporated with $35 \mu \mathrm{g}$ of the Cas9/gRNA targeting vector and ssODN template in $220 \mu \mathrm{L}$ of Opti-MEM (Gibco) using a BTX ECM 2001 system (Harvard Bioscience, Holliston, MA, USA). The electroporated PFFs were cultured at $37^{\circ} \mathrm{C}$ for $72 \mathrm{~h}$ and then trypsinized for genomic DNA extraction. Target sequence regions were amplified by PCR using the following parameters: $94^{\circ} \mathrm{C}$ for $3 \mathrm{~min}$, followed by 35 cycles of $94^{\circ} \mathrm{C}$ for $20 \mathrm{~s}, 60^{\circ} \mathrm{C}$ for $10 \mathrm{~s}$, and $72^{\circ} \mathrm{C}$ for $33 \mathrm{~s}$, with a final extension at $68^{\circ} \mathrm{C}$ for 5 min. For the selection of single-cell colonies, electroporated PFFs were plated onto 10-cm Petri dishes after 48-72 h of culture. Single-cell colonies were picked and cultured in 24-well plates for recovery, while a small part of each colony was lysed for genotyping. The regions surrounding the intended point mutations were amplified and then sequenced. Amplicons with multi-peaks were cloned into the pLB vector (Tiangen, Beijing, China) to identify the exact sequence of each allele.

Somatic cell nuclear transfer.

The SCNT protocol was conducted as described by Lai et al(Fan et al., 2013). Briefly, cumulus-oocyte complexes were matured at $39^{\circ} \mathrm{C}$ for $40 \mathrm{~h}$ in maturation medium after removing the follicles. The first polar body was then aspirated from mature oocytes using a glass pipette, and, finally, the donor cells were fused with enucleated oocytes using BTX electrofusion equipment. The reconstructed embryos were then cultured for nearly $12 \mathrm{~h}$ before being transferred into surrogate sows. Pregnancy was monitored by ultrasonography after 30 days. Piglet DNA was extracted from the tail and analyzed via PCR sequencing. The amplicons were also ligated to the pLB vector to identify the exact sequence of both alleles.

\section{Identification of genetically modified piglets.}

The primers designed for amplification of the COL2A1 -specific Cas9/gRNA plasmid are shown in Figure 1C. PCR was performed using the following parameters: $94^{\circ} \mathrm{C}$ for $5 \mathrm{~min}$, followed by 40 cycles of $94^{\circ} \mathrm{C}$ for 30 $\mathrm{s}, 60^{\circ} \mathrm{C}$ for $30 \mathrm{~s}$, and $72^{\circ} \mathrm{C}$ for $33 \mathrm{~s}$, with a final extension of $72^{\circ} \mathrm{C}$ for $5 \mathrm{~min}$. Genomic DNA from wild-type Bama miniature pigs was used as negative control.

Radiographic analysis.

Autoradiographs of whole-body skeletons and cartilage of piglets were taken on radiographic film (ROTANODE, Tokyo, Japan) using the YEMA Radiography System equipped with a digital camera (Varian, California, USA). The image exposure factors were $30 \mathrm{kV} 2 \mathrm{mAs}$.

Histological and immunohistochemical analysis.

For histological analyses, femurs, humeri, and tracheas were collected from COL2A1 mutant piglets and WT piglets after removing the surrounding muscles and fascia. Femurs, humeri, and tracheas were fixed in $4 \%$ paraformaldehyde at 4degC. The bones were subsequently decalcified in 15\% EDTA for 3 weeks, dehydrated in increasing concentrations of ethanol ( $70 \%$ for $7 \mathrm{~h}, 80 \%$ for $2 \mathrm{~h}, 96 \%$ for $2 \mathrm{~h}$, and $100 \%$ for $4 \mathrm{~h}$ ), washed with xylene, and embedded in paraffin. Then, $5-\mu \mathrm{m}$ thick sections were cut and used for H\&E(Esapa et al., 2012a) and safranin O/fast green staining as previously described(Y. Chen et al., 2017; Schumacher, Joiner, 
Less, Drewry, \& Williams, 2016), and were then analyzed under a microscope (catalog \#BX43, Olympus, Tokyo, Japan). For immunohistochemical analysis, sections were incubated with an anti-COL2A1 primary antibody (catalog \#BA0533; Boster Biological Technology, Wuhan, China) in 3\% BSA overnight at $4^{\circ} \mathrm{C}$. After washing with PBS, the sections were incubated with secondary antibody (catalog \#BA1039; Boster Biological Technology, Wuhan, China) for $30 \mathrm{~min}$, and the staining intensity was analyzed by microscopy.

Western blotting analysis.

Fresh cartilage and trachea samples were ground and then resolved in lysis buffer. The resultant protein samples were quantified using a BCA protein assay kit (Beyotime, Haimen, China). The isolated proteins were separated by SDS-PAGE and then transferred to nitrocellulose membranes. The blots were incubated with a primary antibody against type II collagen (\#BA0533, Boster Biological Technology), washed, and then incubated with a horseradish peroxidase-labeled secondary antibody (catalog \#BA1039; Boster Biological Technology). Finally, the blotting bands were detected with an ECL-Plus western blotting reagent (Beyotime).

Real-time RT-PCR.

Total RNA was extracted from femurs, humeri, and tracheas of newborn WT and COL2A1 KO piglets using TRNzol-A + reagent (Tiangen), treated with DNase I (Fermentas, Massachusetts, USA), and reversetranscribed to cDNA using the BioRT cDNA First-Strand Synthesis Kit (Tiangen). qPCR was performed using the ABI PRISM 7500 FAST Real-Time PCR System (Applied Biosystems, Massachusetts, USA), and the $2^{-\mathrm{Ct}}$ formula was used to determine relative gene expression, which was normalized to the level of GAPDH mRNA. All experiments were repeated three times, and the data are expressed as means $\pm \mathrm{SEM}$.

Off-target analysis.

To test for off-target mutations in the COL2A1 mutant piglets, the top eight POTS were selected for each sgRNA using the CasOFFinder Tool (http://www.rgenome.net/cas-offinder/new). Amplicons were subjected to Sanger sequence. The primers used are listed in Table S1.

Statistical analysis.

A two-tailed Student's $t$-test was used for comparisons between groups, and one-way analysis of variance (ANOVA) with Tukey'spost-hoc test was used for comparisons between multiple groups. Significance compared to untreated controls is denoted with ${ }^{*} P<0.05$, ${ }^{* *} P<0.01$, and ${ }^{*} * * P<0.001$ in the figures and figure legends. Statistical analysis was performed in GraphPad Prism 7.0 (GraphPad Software, La Jolla, CA, USA).

\section{Results}

Generation of a pig model with type II collagen deficiency

CRISPR/Cas9 technology is an effective approach for the generation of double-stranded DNA breaks to facilitate homologous recombination-mediated modification of target genes. We designed two guide RNAs (gRNAs) specific for COL2A1 exon 27 where is highly conserved among different species (Figure 1A), and one single-stranded donor oligonucleotide (ssOND) for insertion into the COL2A1 locusvia homologydirected repair (HDR) (Figure 1B and 1C). One of the target gRNAs was tested to verify that doublestranded DNA breaks could be effectively generated (Figure 1D). Both the Cas9/gRNA expression vector and single-stranded oligonucleotide (ssODN) were transfected into porcine fetal fibroblasts (PFFs). We screened 366 PFFs by PCR and identified 7 positive clones heterozygous for a mutation in exon 27 of the COL2A1 gene. We selected a cell clone that contained a compound heterozygous mutation and obtained 1301 embryos following SCNT. One of the compound COL2A1 variants, c.1744G $>$ A, was previously reported to be associated with ANFH(Kishiya, Nakamura, Ohishi, Furukawa, \& Ishibashi, 2014), while a novel variant, c.1749delA, was predicted to result in a frameshift mutation (Figure 1E). All the embryos were surgically transferred to five surrogate sows, three of which became pregnant. Seven piglets were born by natural delivery. However, the piglets all died shortly after birth, and all carried the compound heterozygous 
mutations identified by PCR, T-cloning and Sanger sequencing. Additionally, the piglets exhibited many of the phenotypical features associated with type II collagenopathies and afforded us an opportunity to establish the cause of the premature death of the animals. As off-target effects are a major concern when using the CRISPR/Cas9 system, we performed Sanger sequencing for eight potential off-target sites (POTS). No off-target effects were found in the COL2A1 mutant piglets (Supporting Figure S1 and Supporting Table $\mathrm{S} 1)$.

\section{Phenotype characterization of COL2A1 knockout pigs}

As shown in Figure 2A, COL2A1 knockout (KO) piglets were shorter than the controls. The flat face, cleft palate, and limb hypoplasia observed in the KO pig are phenotypes typically seen in patients with type II collagenopathy (Figure 2B to 2D)(Zhang et al., 2020). The cleft palate is also considered as a typical phenotype caused by COL2A1 variants in mouse models(Barbieri et al., 2003; Vandenberg et al., 1991). Moreover, the COL2A1 KO piglets had a short nose indicative of mid-face hypoplasia, and the ratio of abdomen circumference to chest circumference suggested that the KO piglets had a protuberant abdomen (Figure 2E).

Radiographic examinations showed that the COL2A1 KO piglets had a markedly underdeveloped skeleton. Lateral radiographic views showed a depressed nasal bridge and short maxilla, which corresponded to the visible flat-face phenotype. Ovoid vertebral bodies could also be observed in the KO pigs (Figure $2 \mathrm{~F}$ ). Radiographic images of the COL2A1 KO pigs further showed thoracic dysplasia, shortened long bones with ragged metaphyses, iliac hypoplasia, and brachydactyly with short metacarpals (Figure 2G to 2I). Figure 2J shows the measured length of the forelimb, hindlimb, spine, and maxilla.

Bone structure is abnormal in COL2A1 KO pigs

For analysis of bone and cartilage pathology, we isolated the long bones and ribs of both KO and wild-type (WT) piglets. We found that the long bones of KO pigs were significantly shorter than those of WT pigs (Figure 3A and 3B). In the COL2A1 KO pigs, the heads of the humerus and femur were underdeveloped, and all the long bone epiphyses were soft and the margins, especially that of the joint structure, were unclear. Interestingly, the ribs of COL2A1 KO pigs were also shorter than those of WT pigs, which resulted in a narrower thoracic cavity (Figure 3C). The lengths of the long bones and ribs are shown in Figure 3D.

\section{Skeleton dysplasia in COL2A1 KO pigs restricts organ development}

To explore whether the skeleton deformities affected organ development, we isolated the organs of both COL2A1 KO and WT pigs (Figure 3E). In the mutant pigs, the absolute weights of all the organs were marginally smaller than those of the controls, and showed no significant differences in morphology (Figure $3 \mathrm{E}$ and $3 \mathrm{G}$ ). However, the weight of the lungs of COL2A1 KO pigs was lower than that of WT pigs, indicating that the deformity of the rib cage restricted lung tissue development (Figure 3F). During the process of isolating the lung, we found that the tracheas of KO pigs were soft and inelastic, a critical factor that prompted us to further explore the cause of death of the COL2A1 mutant pigs shortly after birth.

Histomorphology and pathology of the articular cartilage of the limbs of COL2A1 KO pigs

To understand the pathogenic basis for the skeletal dysplasia phenotype, we investigated the histology of the articular cartilage of the COL2A1 KO pigs (Figure 4). The cartilage of the KO pigs presented porous areas, as well as barely defined superficial, transitional, and deep zones, the three layers that comprise normal articular cartilage. Compared with that of WT piglets (Figure $4 \mathrm{~A}-\alpha, \beta$ ), the femoral head cartilage of KO piglets (Figure 4A- $\gamma$ ) presented vacuolized chondrocytes (arrows) and disrupted collagen formation. Similar changes could be seen in the cartilage of knee joint (Figure 4B). Besides, the lack of regular alignment of hypertrophic chondrocytes was seen in the Figure $4 \mathrm{~A}-\gamma$ and Figure $4 \mathrm{~B}-\gamma$.

Articular cartilage formation in the hip and knee joint was assessed by safranin $\mathrm{O} /$ fast green staining (Figure $5 \mathrm{~A}$ and $5 \mathrm{C}$ ). Safranin $\mathrm{O}$ staining intensity was greatly reduced in the articular cartilage of COL2A1 KO pigs compared with that of WT animals. We also compared the immunoreactivity of type II collagen in the 
articular cartilage of WT and COL2A1 KO pigs by immunohistochemical analysis using sections from the femoral head (Figure 5B) and knee articular cartilage (Figure 5D), and found that the expression of type II collagen was decreased in $\mathrm{KO}$ pigs.

The morphological results of hematoxylin and eosin (H\&E), safranin $\mathrm{O} /$ fast green, and immunohistochemical staining of humerus head and trochlea sections are shown in Supporting Figure S2 and S3. Besides, the thickness of the cartilage in each joint was compared and exhibit in Supporting Figure S4.

\section{Histomorphology and pathology of the tracheal cartilage in COL2A1 KO pigs}

Based on our finding that the tracheas of KO pigs were soft and inelastic, we further investigated the pathological status of the tracheal cartilage. As can be seen in H\&E-stained sections, the tracheal cartilage of COL2A1 KO pigs was flattened and lacked the typical C-shaped curve, and the lumen was essentially obliterated (Grade IV) (Figure 6A). Moreover, the alignment of the pseudostratified ciliated columnar epithelium was lost, and the goblet cells were larger than those of WT pigs (Figure 6A- $\gamma$ ). Although the thickness of the tracheal cartilage was similar between COL2A1 KO and WT piglets, the mutant piglets presented a reduced number of chondrocytes (Figure 6B). Immunohistochemical analysis further indicated that the expression of type II collagen was reduced in the tracheal cartilage of $\mathrm{KO}$ pigs (Figure 6C). The results of the picrosirius red staining demonstrated that there were fewer collagen fibers in the perichondrium and less hyaline cartilage in the trachea of KO pigs than in those of WT pigs (Figure 6D).

Healthy tracheal cartilage helps keep the airways open when breathing; however, lack of type II collagen expression leads to tracheal collapse and blockage of fresh air entry into the lungs, which may have been the cause of the premature death of our COL2A1 KO piglets.

Type II collagen was decreased in the articular cartilage of COL2A1 KO pigs

To confirm that the loss of COL2A1 expression in the KO piglets was indeed due to the mutations generated in the COL2A1 gene, western blot and real-time qPCR was performed for the articular cartilage of the major joints (hip, knee, shoulder, and elbow). As shown in Figure 7A and 7B, the COL2A1 protein expression level was notably reduced in KO pigs compared with that of control pigs, while qPCR analysis indicated that COL2A1 mRNA levels were significantly reduced in the articular and tracheal cartilage of $\mathrm{KO}$ pigs when compared with the levels in WT pigs (Figure 7C to 7E). The predicted 3D structure models suggested that the structure of type II collagen would be disrupted as a result of the c.1749delA mutation in the COL2A1 gene (Figure 7F). The above data indicated that the level of type II collagen was decreased in the mutant piglets, which resulted in underdeveloped cartilage and skeletal deformities.

\section{Discussion}

In this study, we generated genetically modified swine carrying COL2A1 mutations that successfully recapitulated abnormal early bone and cartilage development in humans. Importantly, our swine model provided convincing evidence that tracheal collapse was the most likely cause of the premature death of the COL2A1 mutant piglets that occurred shortly after their birth. Additionally, this is the first genetically modified swine model generated for the investigation of GSDs, which expands the range of applications of genome editing technology in large mammals for human disease modeling.

In our study, a swine model harboring compound heterozygous mutations (c.1744G > A and c.1749delA) in the COL2A1 gene displayed phenotypes typically observed in skeletal dysplasia. The c.1744G $>$ A mutation was previously identified in three generations of a family that all suffered from bilateral hip joint lesions and were diagnosed with ANFH(Kishiya et al., 2014). The severe skeletal defects in our COL2A1 mutant piglets, such as significantly shorter limbs, narrower thoracic cavity, and underdeveloped vertebral body, correspond to the clinical phenotypes of PLSD-T(Nishimura et al., 2004), a rare skeletal dysplasia characterized by extremely short limbs, platyspondyly, and severe pelvic hypoplasia(Nishimura et al., 2004; Zankl et al., 2005). This disorder is usually caused by mutations in the C-propeptide region of COL2A1 and is lethal in the perinatal period in most reported cases(Desir et al., 2012; Nishimura et al., 2004; Okamoto et al., 2012; Zankl et al., 2005). Skeletal disorders similar to those of PLSD-T patients also occurred in a mouse 
model with an ENU-induced missense mutation (c.4406A $>$ C) in the C-propeptide coding region ofCol2a1 (Furuichi et al., 2011). In this mouse model, heterozygotes were smaller than their WT siblings and showed disproportionately short limbs and slightly hypoplastic vertebral bodies, while homozygotes died at birth and exhibited severe dwarfism with shortened limbs and snout. Other mouse models of GSDs have also been established, including for SEDC, osteoarthritis, and disproportionate micromelia(Esapa et al., 2012b; Helminen et al., 1993; Pace et al., 1997).

Histological analysis showed that the COL2A1 KO piglets displayed reduced articular cartilage thickness in the major limb joints compared with that of WT piglets, and the articular cartilage tissue exhibited porous areas and indiscernible superficial, transitional, and proliferative zones. Moreover, the regular alignment of hypertrophic chondrocytes was lost, as has also been reported for Col2a1mutant mice, although porous areas were not seen in the cartilage tissue of these animals(Esapa et al., 2012a; Garofalo et al., 1991; Liang et al., 2014). A previous study suggested that loss of chondrocyte columnar organization was due to the increased deposition of mutated type II collagen in cartilage, as was also seen in other transgenic mouse models of SEDC(Donahue et al., 2003; Gaiser et al., 2002). However, the mechanism involved in the loss of the hypertrophic zone in cartilage has also been reported to result from the activation of the endoplasmic reticulum (ER) stress-unfolding protein response-apoptosis cascade resulting from the accumulation of mutated type II collagen, that finally leads to chondrodysplasia(Chung, Jensen, Gawron, Steplewski, \& Fertala, 2009; Liang et al., 2014). The enlarged and extended chondrocyte phenotype seen in our model is also typically observed in affected human patients with COL2A1 mutations and Col2a1 mutant mice(Esapa et al., 2012a; Su et al., 2010). A mutant form of type II collagen was revealed to lead to articular chondrocyte hypertrophy through the inhibition of integrin beta 1/SMAD1 interaction(Lian et al., 2019). Surprisingly, in the safranin O-/fast green-stained sections of cartilage tissue of the femoral head (Figure 4C), red safranin O staining, which represents cartilage, was absent. We suspect that this was due not only to the COL2A1 mutations leading to reduced cartilage formation, but also the lack of the type II collagen in the cartilage. Other safranin O-/fast green-stained sections also showed reduced safranin O staining intensity (Figure 5A; Supporting Figure S3A and S3C), which has also been reported in previous studies(Fernandes, Seegmiller, Nelson, \& Eyre, 2003). Notably, we did not perform transmission electron microscopy (TEM) analysis as the piglets died too abruptly to enable fresh tissue collection. However, other studies have indicated that the rough ER and Golgi bodies in mutated chondrocytes are dilated, and there is aberrant accumulation of glycogen granules, as well as fewer and thinner collagen fiber aggregates, in the cartilage matrix of Col2a1 mutant mice(Arita et al., 2002; Garofalo et al., 1991; Li et al., 1995).

Importantly, in our study, we detected tracheal collapse inCOL2A1 mutant piglets, which was almost certainly the cause of their death shortly after birth. We found that the tracheal cartilage ofCOL2A1 mutant piglets was flattened, lacking the typical C-shaped curve, and the lumen was essentially obliterated. The picrosirius red-stained sections showed a decreased density of collagen fibers in the mutant piglets (Figure 6D). Additionally, in our COL2A1 mutant pigs, the pseudostratified ciliated columnar epithelium was not aligned, and the goblet cells were larger than those of control piglets (Figure 6A). These findings have not been previously reported. Two patients affected with PLSD-T caused by COL2A1 mutations were reported to suffer only from respiratory failure in infancy(Nishimura et al., 2004; Okamoto et al., 2012), while a few studies have only indicated that transgenic mice with Col2a1 mutations show severe respiratory failure(Barbieri et al., 2003; Donahue et al., 2003). Nevertheless, pulmonary hypoplasia and tracheal collapse are characteristic findings that have been presumed to be the cause of neonatal lethality in atelosteogenesis type 1 (AO1), in which some phenotypes are similar to those observed in PLSD-T(Bejjani et al., 1998).

The pathological mechanism of COL2A1 mutation has been study by lots of research. We have known that COL2A1 gene encodes the alpha 1 chain of procollagen type II, and three alpha 1 chains are folded together in a triple-helical configuration to form the procollagen homotrimer. After secretion into the extracellular matrix, the N- and C-terminal propeptides are removed, forming the mature type II collagen molecule(Bejjani et al., 1998; Strom \& Upholt, 1984). TheCOL2A1 gene comprises 54 exons, with the triple-helical region ranging from exon 8 to exon 51, flanked by $\mathrm{N}$ - and C-propeptide domains(Barat-Houari et al., 2016). Most mutations in the COL2A1 gene occur in the central domain that encodes the triple-helical 
region(Hoornaert et al., 2006); consequently, when mutated, this domain is believed to exert a dominantnegative effect by interfering with the normal assembly of collagen chains, as shown in a Col2a1 mutant (p.R798C) mouse model(Gaiser et al., 2002). Mutations in exon 2, encoding the N-propeptide domain, cause ocular disorders such as Stickler syndrome. COL2A1 mRNA undergoes alternative splicing, resulting in two isoforms. The long form (type IIA isoform) is predominantly expressed by chondroprogenitor cells during early development and in the vitreous of the eye, while the shorter (IIB) form is expressed by differentiating cartilage chondrocytes in adults(McAlinden et al., 2008; Richards \& Snead, 2008). Mutations in exon 2 of COL2A1 can have dramatically different effects on pre-mRNA splicing, and lead to Stickler syndrome or other type II collagenopathies(Richards \& Snead, 2008). Mutations in this exon usually cause a mild disorder, as demonstrated in a transgenic mouse model with a mutated 5' splice site of COL2A1 exon 2; these mice with apparent expression of only the exon 2-containing IIA mRNA isoform displayed no overt phenotype and appeared healthy(Lewis et al., 2012). In contrast, the C-propeptide has been reported to play a crucial role in the formation of the collagen triple-helical structure(Doege \& Fessler, 1986; Khoshnoodi, Cartailler, Alvares, Veis, \& Hudson, 2006). After transfer into the ER, procollagen chains associate viatheir C-propeptides to form homotrimers, and this interaction is stabilized by disulfide bonds.

In summary, using a CRISPR/Cas 9 approach, we have established a pig model carrying compound COL2A1 mutations. This COL2A1 mutant model exhibits overt and typical phenotypes that resemble those of PLSD$\mathrm{T}$, and mainly involve abnormal early cartilage development. Our model paves the way for the establishment of large-animal models to simulate other hereditary skeletal diseases, such as ANFH and OA, and to develop effective prevention and treatment strategies.

\section{Acknowledgements}

The authors are grateful to all the animals in this study. And we thank Jianing Ning, Lin Zhou, Jiahuan Chen, Xiaodong Zou, Lerong Ma and Ang Su for their excellent technical assistance in this study.

\section{Competing interests}

The authors declare no competing interests.

\section{Data availability}

All data needed to evaluate the conclusions in the paper are present in the paper and/or the Supplementary Materials. Additional data related to this paper may be requested from the authors.

\section{References}

Arita, M., Li, S. W., Kopen, G., Adachi, E., Jimenez, S. A., \& Fertala, A. (2002). Skeletal abnormalities and ultrastructural changes of cartilage in transgenic mice expressing a collagen II gene (COL2A1) with a Cys for Arg-alpha1-519 substitution. Osteoarthritis Cartilage, 10 (10), 808-815. Retrieved fromhttps://www.ncbi.nlm.nih.gov/pubmed/12359167

Barat-Houari, M., Sarrabay, G., Gatinois, V., Fabre, A., Dumont, B., Genevieve, D., \& Touitou, I. (2016). Mutation Update for COL2A1 Gene Variants Associated with Type II Collagenopathies. Hum Mutat, 37 (1), 7-15. doi:10.1002/humu.22915

Barbieri, O., Astigiano, S., Morini, M., Tavella, S., Schito, A., Corsi, A., . . . Garofalo, S. (2003). Depletion of cartilage collagen fibrils in mice carrying a dominant negative Col2a1 transgene affects chondrocyte differentiation. Am J Physiol Cell Physiol, 285 (6), C1504-1512. doi:10.1152/ajpcell.00579.2002

Bejjani, B. A., Oberg, K. C., Wilkins, I., Moise, A., Langston, C., Superti-Furga, A., \& Lupski, J. R. (1998). Prenatal ultrasonographic description and postnatal pathological findings in atelosteogenesis type 1 . Am $J$ Med Genet, 79 (5), 392-395. Retrieved fromhttps://www.ncbi.nlm.nih.gov/pubmed/9779808

Chen, C., Jiang, Y., Xu, C., Liu, X., Hu, L., Xiang, Y., . . . Tang, S. (2016). Skeleton Genetics: a comprehensive database for genes and mutations related to genetic skeletal disorders. Database (Oxford), 2016 . doi:10.1093/database/baw127 
Chen, Y., Huang, Y. C., Yan, C. H., Chiu, K. Y., Wei, Q., Zhao, J., . . . Lu, W. W. (2017). Abnormal subchondral bone remodeling and its association with articular cartilage degradation in knees of type 2 diabetes patients. Bone Res, 5 , 17034. doi:10.1038/boneres.2017.34

Chung, H. J., Jensen, D. A., Gawron, K., Steplewski, A., \& Fertala, A. (2009). R992C (p.R1192C) Substitution in collagen II alters the structure of mutant molecules and induces the unfolded protein response. $J$ Mol Biol, 390 (2), 306-318. doi:10.1016/j.jmb.2009.05.004

Cong, L., Ran, F. A., Cox, D., Lin, S., Barretto, R., Habib, N., . . . Zhang, F. (2013). Multiplex genome engineering using CRISPR/Cas systems.Science, 339 (6121), 819-823. doi:10.1126/science.1231143

Deng, H., Huang, X., \& Yuan, L. (2016). Molecular genetics of the COL2A1-related disorders. Mutat Res Rev Mutat Res, 768 , 1-13. doi:10.1016/j.mrrev.2016.02.003

Desir, J., Cassart, M., Donner, C., Coucke, P., Abramowicz, M., \& Mortier, G. (2012). Spondyloperipheral dysplasia as the mosaic form of platyspondylic lethal skeletal dyplasia torrance type in mother and fetus with the same COL2A1 mutation. Am J Med Genet A, 158 A (8), 1948-1952. doi:10.1002/ajmg.a.35301

Doege, K. J., \& Fessler, J. H. (1986). Folding of carboxyl domain and assembly of procollagen I. J Biol Chem, 261 (19), 8924-8935. Retrieved fromhttps://www.ncbi.nlm.nih.gov/pubmed/3722183

Donahue, L. R., Chang, B., Mohan, S., Miyakoshi, N., Wergedal, J. E., Baylink, D. J., . . . Davisson, M. T. (2003). A missense mutation in the mouse Col2a1 gene causes spondyloepiphyseal dysplasia congenita, hearing loss, and retinoschisis. J Bone Miner Res, 18 (9), 1612-1621. doi:10.1359/jbmr.2003.18.9.1612

Esapa, C. T., Hough, T. A., Testori, S., Head, R. A., Crane, E. A., Chan, C. P., . . . Thakker, R. V. (2012a). A mouse model for spondyloepiphyseal dysplasia congenita with secondary osteoarthritis due to a Col2a1 mutation. J Bone Miner Res, 27 (2), 413-428. doi:10.1002/jbmr.547

Esapa, C. T., Hough, T. A., Testori, S., Head, R. A., Crane, E. A., Chan, C. P. S., . . . Thakker, R. V. (2012b). A mouse model for spondyloepiphyseal dysplasia congenita with secondary osteoarthritis due to a Col2a1 mutation. Journal of Bone and Mineral Research, 27 (2), 413-428. doi:10.1002/jbmr.547

Fan, N., Chen, J., Shang, Z., Dou, H., Ji, G., Zou, Q., . . . Lai, L. (2013). Piglets cloned from induced pluripotent stem cells. Cell Res, 23 (1), 162-166. doi:10.1038/cr.2012.176

Fernandes, R. J., Seegmiller, R. E., Nelson, W. R., \& Eyre, D. R. (2003). Protein consequences of the Col2a1 C-propeptide mutation in the chondrodysplastic Dmm mouse. Matrix Biol, 22 (5), 449-453. Retrieved fromhttps://www.ncbi.nlm.nih.gov/pubmed/14614991

Furuichi, T., Masuya, H., Murakami, T., Nishida, K., Nishimura, G., Suzuki, T., . . . Ikegawa, S. (2011). ENU-induced missense mutation in the C-propeptide coding region of Col2a1 creates a mouse model of platyspondylic lethal skeletal dysplasia, Torrance type. Mamm Genome, 22 (5-6), 318-328. doi:10.1007/s00335-011-9329-3

Gaiser, K. G., Maddox, B. K., Bann, J. G., Boswell, B. A., Keene, D. R., Garofalo, S., \& Horton, W. A. (2002). Y-position collagen II mutation disrupts cartilage formation and skeletal development in a transgenic mouse model of spondyloepiphyseal dysplasia. J Bone Miner Res, 17 (1), 39-47. doi:10.1359/jbmr.2002.17.1.39

Garofalo, S., Vuorio, E., Metsaranta, M., Rosati, R., Toman, D., Vaughan, J., . . . et al. (1991). Reduced amounts of cartilage collagen fibrils and growth plate anomalies in transgenic mice harboring a glycine-tocysteine mutation in the mouse type II procollagen alpha 1-chain gene. Proc Natl Acad Sci U S A, 88 (21), 9648-9652. doi:10.1073/pnas.88.21.9648

Han, K., Liang, L., Li, L., Ouyang, Z., Zhao, B., Wang, Q., . . . Fan, N. (2017). Generation of Hoxc13 knockout pigs recapitulates human ectodermal dysplasia-9. Hum Mol Genet, 26 (1), 184-191. doi:10.1093/hmg/ddw378 
Helminen, H. J., Kiraly, K., Pelttari, A., Tammi, M. I., Vandenberg, P., Pereira, R., . . . et al. (1993). An inbred line of transgenic mice expressing an internally deleted gene for type II procollagen (COL2A1). Young mice have a variable phenotype of a chondrodysplasia and older mice have osteoarthritic changes in joints. J Clin Invest, 92 (2), 582-595. doi:10.1172/JCI116625

Holm, I. E., Alstrup, A. K., \& Luo, Y. (2016). Genetically modified pig models for neurodegenerative disorders. J Pathol, 238 (2), 267-287. doi:10.1002/path.4654

Hoornaert, K. P., Dewinter, C., Vereecke, I., Beemer, F. A., Courtens, W., Fryer, A., . . . Mortier, G. R. (2006). The phenotypic spectrum in patients with arginine to cysteine mutations in the COL2A1 gene. $J$ Med Genet, 43 (5), 406-413. doi:10.1136/jmg.2005.035717

Kaarniranta, K., Ihanamaki, T., Sahlman, J., Pulkkinen, H., Uusitalo, H., Arita, M., . . . Helminen, H. J. (2006). A mouse model for Stickler's syndrome: ocular phenotype of mice carrying a targeted heterozygous inactivation of type II (pro)collagen gene (Col2a1).Exp Eye Res, 83 (2), 297-303. doi:10.1016/j.exer.2005.11.027

Kannu, P., Bateman, J., \& Savarirayan, R. (2012). Clinical phenotypes associated with type II collagen mutations. J Paediatr Child Health, 48 (2), E38-43. doi:10.1111/j.1440-1754.2010.01979.x

Khoshnoodi, J., Cartailler, J. P., Alvares, K., Veis, A., \& Hudson, B. G. (2006). Molecular recognition in the assembly of collagens: terminal noncollagenous domains are key recognition modules in the formation of triple helical protomers. J Biol Chem, 281 (50), 38117-38121. doi:10.1074/jbc.R600025200

Kishiya, M., Nakamura, Y., Ohishi, H., Furukawa, K., \& Ishibashi, Y. (2014). Identification of a novel COL2A1 mutation (c.1744G>A) in a Japanese family: a case report. J Med Case Rep, 8 , 276. doi:10.1186/1752-1947-8-276

Kornak, U., \& Mundlos, S. (2003). Genetic disorders of the skeleton: a developmental approach. Am J Hum Genet, 73 (3), 447-474. doi:10.1086/377110

Lewis, R., Ravindran, S., Wirthlin, L., Traeger, G., Fernandes, R. J., \& McAlinden, A. (2012). Disruption of the developmentally-regulated Col2a1 pre-mRNA alternative splicing switch in a transgenic knock-in mouse model. Matrix Biol, 31 (3), 214-226. doi:10.1016/j.matbio.2011.12.004

Li, S. W., Prockop, D. J., Helminen, H., Fassler, R., Lapvetelainen, T., Kiraly, K., . . . et al. (1995). Transgenic mice with targeted inactivation of the Col2 alpha 1 gene for collagen II develop a skeleton with membranous and periosteal bone but no endochondral bone.Genes Dev, 9 (22), 2821-2830. doi:10.1101/gad.9.22.2821

Lian, C., Wang, X., Qiu, X., Wu, Z., Gao, B., Liu, L., . . . Su, P. (2019). Collagen type II suppresses articular chondrocyte hypertrophy and osteoarthritis progression by promoting integrin beta1-SMAD1 interaction. Bone Res, 7 , 8. doi:10.1038/s41413-019-0046-y

Liang, G., Lian, C., Huang, D., Gao, W., Liang, A., Peng, Y., . . . Huang, D. (2014). Endoplasmic reticulum stress-unfolding protein response-apoptosis cascade causes chondrodysplasia in a col2a1 p.Gly1170Ser mutated mouse model. PLoS One, 9 (1), e86894. doi:10.1371/journal.pone.0086894

Macdonald, D. W., Squires, R. S., Avery, S. A., Adams, J., Baker, M., Cunningham, C. R., . . . Seegmiller, R. E. (2013). Structural Variations in Articular Cartilage Matrix Are Associated with Early-Onset Osteoarthritis in the Spondyloepiphyseal Dysplasia Congenita (Sedc) Mouse. International Journal of Molecular Sciences, 14 (8), 16515-16531. doi:10.3390/ijms140816515

Maddox, B. K., Garofalo, S., Smith, C., Keene, D. R., \& Horton, W. A. (1997). Skeletal development in transgenic mice expressing a mutation at Gly574Ser of type II collagen. Dev Dyn, 208 (2), 170-177. doi:10.1002/(SICI)1097-0177(199702)208:2<170::AID-AJA4>3.0.CO;2-F

McAlinden, A., Majava, M., Bishop, P. N., Perveen, R., Black, G. C., Pierpont, M. E., . . . Mannikko, M. (2008). Missense and nonsense mutations in the alternatively-spliced exon 2 of COL2A1 cause the ocular 
variant of Stickler syndrome. Hum Mutat, 29 (1), 83-90. doi:10.1002/humu.20603

Metsaranta, M., Garofalo, S., Decker, G., Rintala, M., de Crombrugghe, B., \& Vuorio, E. (1992). Chondrodysplasia in transgenic mice harboring a 15-amino acid deletion in the triple helical domain of pro alpha 1(II) collagen chain. J Cell Biol, 118 (1), 203-212. doi:10.1083/jcb.118.1.203

Mortier, G. R., Cohn, D. H., Cormier-Daire, V., Hall, C., Krakow, D., Mundlos, S., . . . Warman, M. L. (2019). Nosology and classification of genetic skeletal disorders: 2019 revision. Am J Med Genet A. doi:10.1002/ajmg.a.61366

Myllyharju, J., \& Kivirikko, K. I. (2004). Collagens, modifying enzymes and their mutations in humans, flies and worms. Trends Genet, 20 (1), 33-43. doi:10.1016/j.tig.2003.11.004

Nishimura, G., Nakashima, E., Mabuchi, A., Shimamoto, K., Shimamoto, T., Shimao, Y., . . . Ikegawa, S. (2004). Identification of COL2A1 mutations in platyspondylic skeletal dysplasia, Torrance type. $J$ Med Genet, 41 (1), 75-79. doi:10.1136/jmg.2003.013722

Okamoto, T., Nagaya, K., Asai, H., Tsuchida, E., Nohara, F., Hayashi, T., . . . Azuma, H. (2012). Platyspondylic lethal dysplasia torrance type with a heterozygous mutation in the triple helical domain of COL2A1 in two sibs from phenotypically normal parents. Am J Med Genet A, 158A (8), 1953-1956. doi:10.1002/ajmg.a.35509

Pace, J. M., Li, Y., Seegmiller, R. E., Teuscher, C., Taylor, B. A., \& Olsen, B. R. (1997). Disproportionate micromelia (Dmm) in mice caused by a mutation in the C-propeptide coding region of Col2a1. Dev Dyn, 208 (1), 25-33. doi:10.1002/(SICI)1097-0177(199701)208:1<25::AID-AJA3>3.0.CO;2-3

Prather, R. S., Lorson, M., Ross, J. W., Whyte, J. J., \& Walters, E. (2013). Genetically engineered pig models for human diseases. Annu Rev Anim Biosci, 1 , 203-219. doi:10.1146/annurev-animal-031412-103715

Richards, A. J., \& Snead, M. P. (2008). The influence of pre-mRNA splicing on phenotypic modification in Stickler's syndrome and other type II collagenopathies. Eye (Lond), 22 (10), 1243-1250. doi:10.1038/eye.2008.34

Schumacher, C. A., Joiner, D. M., Less, K. D., Drewry, M. O., \& Williams, B. O. (2016). Characterization of genetically engineered mouse models carrying Col2a1-cre-induced deletions of Lrp5 and/or Lrp6.Bone Res, 4 , 15042. doi:10.1038/boneres.2015.42

Strom, C. M., \& Upholt, W. B. (1984). Isolation and characterization of genomic clones corresponding to the human type II procollagen gene.Nucleic Acids Res, 12 (2), 1025-1038. doi:10.1093/nar/12.2.1025

Su, P., Zhang, L., Peng, Y., Liang, A., Du, K., \& Huang, D. (2010). A histological and ultrastructural study of femoral head cartilage in a new type II collagenopathy. Int Orthop, 34 (8), 1333-1339. doi:10.1007/s00264010-0985-9

Vandenberg, P., Khillan, J. S., Prockop, D. J., Helminen, H., Kontusaari, S., \& Ala-Kokko, L. (1991). Expression of a partially deleted gene of human type II procollagen (COL2A1) in transgenic mice produces a chondrodysplasia. Proc Natl Acad Sci US A, 88 (17), 7640-7644. doi:10.1073/pnas.88.17.7640

Yan, S., Tu, Z., Liu, Z., Fan, N., Yang, H., Yang, S., . . . Li, X. J. (2018). A Huntingtin Knockin Pig Model Recapitulates Features of Selective Neurodegeneration in Huntington's Disease. Cell, 173 (4), 989-1002 e1013. doi:10.1016/j.cell.2018.03.005

Yang, S., Chang, R., Yang, H., Zhao, T., Hong, Y., Kong, H. E., . . . Li, X. J. (2017). CRISPR/Cas9mediated gene editing ameliorates neurotoxicity in mouse model of Huntington's disease. J Clin Invest, 127 (7), 2719-2724. doi:10.1172/JCI92087

Zankl, A., Neumann, L., Ignatius, J., Nikkels, P., Schrander-Stumpel, C., Mortier, G., . . . Superti-Furga, A. (2005). Dominant negative mutations in the C-propeptide of COL2A1 cause platyspondylic lethal skeletal 
dysplasia, torrance type, and define a novel subfamily within the type 2 collagenopathies. Am J Med Genet A, 133 A (1), 61-67. doi:10.1002/ajmg.a.30531

Zhang, B., Zhang, Y., Wu, N., Li, J., Liu, H., \& Wang, J. (2020). Integrated analysis of COL2A1 variant data and classification of type II collagenopathies. Clin Genet, 97 (3), 383-395. doi:10.1111/cge.13680

Zhou, X., Xin, J., Fan, N., Zou, Q., Huang, J., Ouyang, Z., . . . Lai, L. (2015). Generation of CRISPR/Cas9-mediated gene-targeted pigs via somatic cell nuclear transfer. Cell Mol Life Sci, 72 (6), 1175-1184. doi:10.1007/s00018-014-1744-7

\section{Figure Legends}

Figure 1. Generation of COL2A1 knockout (KO) pigs.(A) The target position of amino acid for mutation are highly conserved among different species. (B) Schematic diagram of the strategy used to generate COL2A1 KO pigs viassODN-mediated homology-directed repair. Two gRNAs were used to target the pig COL2A1 exon 27 to promote DNA breaks and homologous repair. (C) Target sequences of the two sgRNAs and complementary oligo sgRNAs. (D) Sequencing chromatogram showing a DNA break in exon 27. The arrow indicates the Cas9 cleavage site.(E) Sequencing chromatogram showing the compound heterozygous mutation of one of the cell clones selected for SCNT.

Figure 2. Phenotypic characterization and skeletal radiographs of the COL2A1 knockout (KO) piglets. (A) Whole-body photographs of a symptomatic COL2A1 KO piglet and a wild-type (WT) piglet shortly after birth. (B) The underdeveloped nose of the COL2A1 KO pig compared with that of the WT pig. (C)Cleft palate of the KO pig compared with that of the WT pig.(D) Comparison of the fore- and hindlimbs between $\mathrm{KO}$ and WT piglets. (E) Comparison of average body weight, body length, length of the nose, and ratio of chest circumference to abdominal circumference. (F) Autoradiographs showing the abnormal systemic skeletal development in the COL2A1 KO pig. A depressed nasal bridge and short maxilla were noted (yellow arrow). Additionally, lateral radiographic views showed the presence of ovoid vertebral bodies in the KO pigs (blue arrow). (G) Thinner and shorter ribs, smaller and splayed scapulae (yellow arrow), and underdeveloped vertebrae (blue arrow) in KO piglets compared with the same structures in WT piglets. (H) The underdeveloped skeleton of the forelimb in the KO pig compared with that in the WT pig. Shortened long bones with ragged metaphyses (yellow arrow) and brachydactyly with short metacarpals (blue arrow) can be observed in the KO pigs. (I)The underdeveloped skeleton of the hindlimbs of KO piglets compared with those of WT piglets. Iliac hypoplasia (yellow arrow), ragged metaphyses (blue arrow), and brachydactyly with short metacarpals (green arrow) were noted in KO piglets. (J) Comparison of the average length of the forelimbs, hindlimbs, maxilla, mandible, and spine between WT and KO piglets shortly after birth. White bar, $2 \mathrm{~cm}$. Data are presented as means \pm SEM. ${ }^{*} P<0.05,{ }^{* *} P<0.01,{ }^{* * *} P<0.001$.

Figure 3. Abnormal gross morphology of the bones and organs in COL2A1 knockout (KO) pigs compared with those of wild-type (WT) pigs. (A) Gross morphology of forelimb bones showing the greatly reduced lengths of the long bones and unclear margins of the epiphyses in the KO pigs; humerus, combined ulna and radius, and forehoof. (B) Gross morphology of hindlimb bones demonstrating the significantly shorter long bones and deformities in the hindlimbs of the KO pigs; femur, tibia, and combined fibula and hindhoof.(C) Gross morphology of the rib cage of COL2A1 KO pigs showing a notable difference in size, indicating that the $\mathrm{KO}$ pigs have a narrower thoracic cavity. (D) Comparison of the average length of the WT and KO piglets. All the bones of the KO pigs were shorter than those of the WT pigs. (E) No obvious differences could be seen in the sizes and shapes of the organs (liver, heart, spleen, kidney, and stomach). (F) Comparison of the lungs showed that KO pigs presented smaller lungs and a thinner trachea (arrow). (G) The absolute weights of all the organs were marginally lower in the KO pigs. Lung volume and weight were significantly decreased in the KO pigs. White bar, $2 \mathrm{~cm}$. Data are presented as means $\pm \mathrm{SEM}$. ${ }^{*} P<0.05,{ }^{* *} P<0.01$, ${ }^{* * *} P<0.001 ;$ ns, not significant.

Figure 4. Histomorphology and pathology of hindlimb articular cartilage in COL2A1 knockout (KO) pigs. (A) Hematoxylin and eosin (H\&E)-stained sections of the femoral head. The boxed regions (A- $\alpha$ and A- $\beta$ ) represent the superficial, transitional, and deep zones of the articular cartilage in wild-type (WT) pigs. The 
A- $\gamma$ region represents the corresponding abnormal cartilage zones of COL2A1KO pigs, characterized by porous areas with enlarged and vacuolized chondrocytes (arrows). (B) H\&E-stained sections of the knee joint. The boxed regions (B- $\alpha$ and B- $\beta$ ) represent the superficial, transitional, and deep zones of the articular cartilage in WT pigs. The B- $\gamma$ boxed region shows the disordered cartilage zones of the knee joint of the $\mathrm{KO}$ pig, characterized by porous areas with enlarged and vacuolized chondrocytes (arrows). Black bar, 500 $\mu \mathrm{m}$; red bar, $200 \mu \mathrm{m}$; green bar, $100 \mu \mathrm{m}$; blue bar, $50 \mu \mathrm{m}$.

Figure 5. Safranin O- and fast green-stained and immunohistochemical sections of articular cartilage of hindlimbs.(A) Safranin O- and fast green-stained sections showing femoral head articular cartilage morphology, demonstrating the lack of cartilage tissue formation in COL2A1 KO pigs. (B)Immunohistochemical staining showing that the expression of type II collagen was lower in the femoral head of KO pigs than in that of WT pigs. (C) Safranin O- and fast green-stained sections showing knee articular cartilage morphology, demonstrating the lack of cartilage tissue formation in COL2A1 KO pigs.(D) Immunohistochemical staining showing the decreased expression of type II collagen in the knee joints of KO pigs. Black bar, 500 $\mu \mathrm{m}$; red bar, $200 \mu \mathrm{m}$; green bar, $100 \mu \mathrm{m}$; blue bar, $50 \mu \mathrm{m}$.

Figure 6. Histomorphology and pathology of the tracheal cartilage in COL2A1 knockout (KO) pigs. (A) Hematoxylin and eosin (H\&E)-stained tracheal sections. The results of the H\&E staining demonstrated that the trachea of the KO pigs had collapsed, and the lumen was essentially obliterated. The A- $\gamma$ boxed region shows the misaligned pseudostratified ciliated columnar epithelium of the COL2A1 KO pigs compared with that of WT pigs (A- $\alpha)$. Furthermore, the A- $\beta$ boxed region (WT pigs) shows stronger staining of the tracheal cartilage and a more uniform texture than that of the cartilage shown in the A- $\delta$ region (KO pigs). (B) The thickness of the tracheal cartilage in the KO pigs was comparable with that of WT pigs; however, there were fewer chondrocytes in the tracheal cartilage of the KO pigs.(C) Immunohistochemical staining showing that type II collagen expression in tracheal cartilage was decreased in KO pigs compared with that in WT pigs. (D) Picrosirius red staining demonstrating that there were fewer collagen fibers in the perichondrium and less hyaline cartilage in the trachea of KO pigs when compared with those of WT pigs. Black bar, $500 \mu \mathrm{m}$; red bar, $200 \mu \mathrm{m}$; green bar, $100 \mu \mathrm{m}$; blue bar, $50 \mu \mathrm{m}$. Data are presented as means \pm SEM. ${ }^{*} P<0.05 ;{ }^{* *} P$ $<0.01 ;{ }^{* * *} P<0.001 ;$ ns, not significant.

Figure 7. Western blot and qPCR analyses of COL2A1 expression levels. (A) Western blots of COL2A1 in cartilage tissues of the hip, knee, and ankle joints from three KO piglets (F0-1, F0-3, and F0-6). (B) Western blots of COL2A1 in cartilage tissues of the shoulder, elbow, and wrist joints from three KO piglets (F0-1, F0-3, and F0-6). (C-E) Relative COL2A1 mRNA expression levels in hindlimb joints, forelimb joints, and tracheal cartilage as quantified by qPCR. (F) Computer modeling of the COL2A1 3D structure and impact of the COL2A1 mono-allelic targeted mutations. Data are presented as means \pm SEM. ${ }^{*} P<0.05 ;{ }^{* *} P<$ $0.01 ;{ }^{* * *} P<0.001$.

\section{Hosted file}

Figure 1.tif available at https://authorea.com/users/302123/articles/432136-a-crisprengineered-swine-model-of-col2a1-deficiency-recapitulates-altered-early-skeletaldevelopmental-defects-in-humans

\section{Hosted file}

Figure 2.tif available at https://authorea.com/users/302123/articles/432136-a-crisprengineered-swine-model-of-col2a1-deficiency-recapitulates-altered-early-skeletaldevelopmental-defects-in-humans

\section{Hosted file}

Figure 3.tif available at https://authorea.com/users/302123/articles/432136-a-crisprengineered-swine-model-of-col2a1-deficiency-recapitulates-altered-early-skeletaldevelopmental-defects-in-humans 


\section{Hosted file}

Figure 4.tif available at https://authorea.com/users/302123/articles/432136-a-crisprengineered-swine-model-of-col2a1-deficiency-recapitulates-altered-early-skeletaldevelopmental-defects-in-humans

\section{Hosted file}

Figure 5.tif available at https://authorea.com/users/302123/articles/432136-a-crisprengineered-swine-model-of-col2a1-deficiency-recapitulates-altered-early-skeletaldevelopmental-defects-in-humans

\section{Hosted file}

Figure 6.tif available at https://authorea.com/users/302123/articles/432136-a-crisprengineered-swine-model-of-col2a1-deficiency-recapitulates-altered-early-skeletaldevelopmental-defects-in-humans

\section{Hosted file}

Figure 7.tif available at https://authorea.com/users/302123/articles/432136-a-crisprengineered-swine-model-of-col2a1-deficiency-recapitulates-altered-early-skeletaldevelopmental-defects-in-humans 